\title{
PERCEPCIÓN DE LA CULTURA DE LA SEGURIDAD DE LOS PACIENTES POR PERSONAL DE ENFERMERÍA
}

\section{NURSING PERCEPTION OF PATIENT SAFETY CULTURE}

\author{
Hortensia Castañeda-Hidalgo * \\ Rosalinda Garza Hernández ** \\ Juana Fernanda GonZález Salinas ${ }^{* * *}$ \\ Mercedes Pineda Zúñiga ${ }^{* * *}$ \\ Gloria Acevedo Porras ${ }^{* * * *}$ \\ Angélica Aguilera Pérez ${ }^{* * * * *}$
}

\begin{abstract}
RESUMEN
Objetivo: Identificar las áreas de fortaleza y oportunidad de mejora continua, percibidas por el personal de enfermería en relación a la cultura de seguridad en la atención de los pacientes en un Hospital General del Sistema de Salud, en Tampico, Tamaulipas, México. Material y método: estudio cuantitativo, descriptivo con diseño transversal, realizado en un hospital de segundo nivel de atención. Muestra fue de 195 enfermeras, obtenida en un muestreo probabilístico por turnos. La recolección de los datos para el análisis de la cultura de la seguridad fue a través de un indicador tipo encuesta de la Agency for Healthcare Research and Quality-AHQR, validada al contexto de español como Cuestionario sobre seguridad de los pacientes, versión española del Hospital Survey on Patient Safety. Resultados: Las dimensiones percibidas como fortalezas son el trabajo en equipo en la unidad/ servicio y aprendizaje organizacional. Las dimensiones percibidas hacia la mejora continua fueron: dotación de personal, respuesta no punitiva a los errores, apoyo de la gerencia del hospital en la seguridad del paciente, percepción de seguridad, problemas en cambios de turno y transiciones entre servicios/unidades y franqueza de la comunicación. El clima de seguridad fue evaluado con una media de 7,35. El mayor porcentaje no reporta eventos adversos asociados a la atención para la salud. Conclusión: el indicador utilizado permitió identificar la percepción global del personal de enfermería hacia la seguridad en la atención del paciente, identificando seis dimensiones problemáticas con oportunidad hacia la mejora continua; así como dos dimensiones fuertes dentro de la cultura de seguridad.
\end{abstract}

Palabras clave: Seguridad del paciente, enfermeras, mejoramiento de la calidad continúa.

\begin{abstract}
The aim of the study was to identify areas of strength and opportunity for continuous improvement, as perceived by the nursing staff in relation to safety culture in the care of patients in a General Hospital Health System, in Tampico, Tamaulipas, México. Method: The study was a quantitative and descriptive cross-sectional design, made in a secondary care hospital. The sample consisted of 195 nurses on probability sampling by shifts. The data collection for the analysis of safety culture was through a survey type indicator: Agency for Healthcare Research and Quality-AHQR, validated to the Spanish context as Questionnaire on patient safety: Spanish

\footnotetext{
*Enfermera. Profesora Facultad Enfermería-Tampico. UAT, Tamaulipas. México. Email: hcastane@uat.edu.mx

** Enfermera. Profesora Facultad Enfermería-Tampico UAT, Tamaulipas. México. Email: rgarzah@uat.edu.mx

${ }^{* * *}$ Enfermera. Profesora Facultad Enfermería-Tampico UAT, Tamaulipas. México. Email: fgonzale@uat.edu.mx

${ }^{* * *}$ Enfermera. Profesora. Facultad Enfermería-Tampico UAT, Tamaulipas. México. Email: mpineda@uat.edu.mx

${ }^{* * * * *}$ Enfermera. Profesora Facultad Enfermería-Tampico UAT, Tamaulipas. México. Email: gacevedo@uat.edu.mx

${ }^{* * * * * *}$ Enfermera. Profesora Facultad Enfermería-Tampico UAT, Tamaulipas. México. Email: aguilera@uat.edu.mx
} 
version of the Hospital Survey on Patient Safety. Results: The dimensions perceived as strengths are teamwork in the unit/service and organizational learning. The dimensions perceived to continuous improvement were: staffing, no punitive response to errors, management support hospital patient safety, perceived safety, problems with shift changes and transitions between departments/units and openness of communication. Safety climate was assessed with an average of 7.35. The highest percentage reported no adverse events associated with health care. Conclusions: The indicator used identified the global perception of the nursing staff to safety in patient care, identifying six dimensions problem with opportunity for continuous improvement, as well as two strong dimensions within the safety culture.

Key words: Patient safety culture, nurses, quality improvement.

Fecha recepción: 09/07/12 Fecha aceptación: 17/07/13

\section{INTRODUCCIÓN}

El extraordinario desarrollo de la atención sanitaria en los últimos 20 años ha permitido restablecer la salud en situaciones antes impensadas, pero al mismo tiempo su gran complejidad tecnológica y la interacción entre diferentes actores suponen una situación de mayor riesgo para los pacientes (1). Los ciudadanos ayudados por las tecnologías de la información han ido adquiriendo conocimientos sobre las enfermedades y los posibles tratamientos, llegando a cuestionar más que nunca la actuación de los profesionales sanitarios, que en muchas ocasiones no son capaces de responder a este nuevo reto (2).

En el cuidado de la salud asegurar la calidad implica procesos que garanticen que la atención clínica se ajuste a los criterios o normas establecidos. Uno de los principios básicos de la calidad es la prevención y la mejora continua. Esto significa que la calidad es un proceso dinámico y un proyecto interminable, cuyo objetivo es detectar disfunciones tan rápido como sea posible una vez que ocurran. Por lo tanto gestionar la calidad en salud requiere de aplicar principios fundamentales como son: prácticas centradas en el paciente, mejora continua con implicación y liderazgo del equipo directivo y participación de todo el equipo de trabajo; identificación de procesos claves y superar las barreras existentes; toma de decisiones basadas en evidencias con dominio de la información (3).

La seguridad del paciente desde la perspectiva de la atención de salud supone un conjunto de normas, procedimientos, instrumentos y métodos basados en evidencias científicas destinados a minimizar el riesgo de daño sobre agregado y de eventos adversos en la atención de la salud. Incluye medidas que garantizan prácticas diagnósticas, terapéuticas y de cuidados de enfermería seguros, así como de ajuste del medio ambiente, organización y funcionamiento institucional, incluyendo la dotación, competencias y sostenimiento del personal (4).

El informe de la Organización Mundial de la Salud (OMS) en el 2001, en relación a la calidad de la atención y seguridad del paciente establece que: "Las intervenciones de atención de salud se realizan con el propósito de beneficiar al paciente pero también pueden causarles daño. La combinación compleja de procesos, tecnología e interacciones humanas, que constituyen el sistema moderno de prestación de atención de salud, pueden aportar beneficios importantes. Sin embargo, también conlleva un riesgo inevitable de que ocurran eventos adversos" (5).

En los años cincuenta del siglo XX se publicaron los primeros estudios sobre los riesgos de la hospitalización y los errores médicos. La contribución de estos estudios y otros posteriores permitió la implementación de prácticas seguras que han logrado reducir de 
forma extraordinaria la muerte relacionada con eventos adversos en los últimos 25 años. $\mathrm{Al}$ mismo tiempo, los estudios epidemiológicos han contribuido de forma especial al conocimiento sobre la frecuencia del daño asociado a la atención sanitaria. Schimmel, en 1964 (6), llamó la atención tempranamente sobre los riesgos de la hospitalización y Brennan, en 1984 (7), aportó una metodología para el estudio de los eventos adversos que se replicaría en todo el mundo. Gracias a estos estudios hoy se conoce mejor la frecuencia de los eventos adversos y sus determinantes (8).

En 1999, el informe del Instituto de Medicina de EE.UU. "Errar es de humanos: construyendo un sistema más seguro de salud" marca un antes y un después en la concienciación sobre este tema y ha hecho que la investigación sobre seguridad del paciente y las iniciativas para actuar sobre los riesgos evitables sean una prioridad para todas las autoridades sanitarias. Este estudio estimó que los errores clínicos prevenibles son responsables de entre 44.000 y 98.000 muertes de pacientes en hospitales por año. El costo de los errores va desde 17 a 29 millones de dólares anualmente y los costos emocionales relacionados para los pacientes y familiares, así como de sus cuidadores, son incalculables $(9,10)$.

En marzo de 2002 la OMS en su 55a Asamblea Mundial informó tasas muy altas de eventos adversos para diferentes países desarrollados que oscilaron entre 3,2 y $16,6 \%$, corroborando el gran problema existente en el ámbito mundial. Si se adiciona a las miles de personas que como producto del error no murieron pero que quedaron con una discapacidad transitoria o permanente, el problema toma dimensiones casi epidémicas, por lo cual la OMS lanzó la Alianza Mundial para la Seguridad Clínica del Paciente $(11,12)$.

Una cultura positiva sobre seguridad del paciente en las instituciones de salud se perfila como uno de los requisitos esenciales para gestionar el riesgo inherente a las prácticas de atención de salud, con el aprendizaje de los errores y el rediseño de los procesos para evitar que vuelvan a producirse. En este sentido, conseguir una adecuada cultura sobre la seguridad del paciente ha sido señalada como la primera de las "buenas prácticas" o recomendaciones para mejorar la seguridad del paciente en el informe publicado el 2003 por el National Quality Forum de los Estados Unidos (13), en cuya última actualización (14) se indica, además, la medición de la cultura de seguridad, feed-back de los resultados, y las intervenciones consecuentes, como uno de los elementos necesarios para llevar a cabo esta recomendación $(15,16)$.

Cuando la cultura de seguridad es aplicada al cuidado de la salud tiene aún mayor importancia, ya que la seguridad no sólo aplica para la fuerza de trabajo sino también para pacientes que podrían ser dañados por las acciones del personal. La tradicional cultura de seguridad del paciente "culpa y vergüenza" en organizaciones del cuidado de la salud ha sido criticada por obstruir la posibilidad de "aprender de los errores" y ser responsable de causar errores clínicos. Hay un reconocimiento creciente de la necesidad de trasformar la cultura organizacional del cuidado de la salud (17-21).

Helmreich define a la cultura organizacional, como "una compleja red de actitudes nacionales, organizacionales, profesionales y los valores en base a los cuales funcionan los individuos y grupos". Con frecuencia la cultura organizacional se compara de manera figurada como el pegamento que mantiene unida a la organización y, por lo tanto, se asume que dicha cultura es un componente importante y contribuye al desempeño de la organización en su conjunto, a la socialización de los trabajadores, de tal manera que estos aumentan su compromiso con los objetivos de la organización (22).

La cultura de seguridad de acuerdo a la Agency for Healthcare Research and Quality-AHQR, se define como el resultado de los valores individuales y de grupo, actitudes, percepciones, competencias y patrones de comportamiento que determinan el com- 
promiso, el estilo y la competencia de una organización hacia la salud, y el manejo de la seguridad, la cual se caracteriza por una comunicación fundada en la confianza mutua, producto de las percepciones compartidas acerca de la importancia de la seguridad, y por la confianza depositada en la eficacia de las medidas preventivas implementadas para evitar los eventos adversos (23).

El personal de enfermería, como miembro del equipo de salud, debe desarrollar una cultura de calidad e incorporarse a los programas con una actitud proactiva. La calidad en la atención de enfermería es un concepto complejo que comprende la asistencia acorde con el avance de la ciencia, a fin de implementar cuidados que satisfagan las necesidades de cada uno de los pacientes y asegurar su continuidad $(24,25)$. Siendo el grupo más grande de los proveedores del cuidado de la salud, constituyen una parte indispensable de los equipos multidisciplinarios que están encontrando soluciones innovadoras para mejorar la seguridad en beneficio del paciente. Una aproximación de los sistemas toma una perspectiva más amplia al buscar soluciones en el ambiente físico y cultural para dar cuidado seguro de alta calidad (26).

Actualmente analizar la cultura de la seguridad en la atención de los pacientes dentro de los sistemas de salud, constituye un pilar fundamental, para implementar y arraigar en la organización el valor de la seguridad, permite identificar aéreas de fortalezas y oportunidades hacia la mejora continua que permitan establecer un plan de intervenciones, que guíen a establecer cuidados seguros $y$ de calidad en beneficio de los pacientes y sus familias y con ello, minimizar las repercusiones de eventos adversos que dañan la integridad física, emocional y económica de los implicados. Con el aumento del énfasis en el propósito de la seguridad en las organizaciones de cuidado de la salud, crear una cultura de seguridad del paciente se vuelve extremadamente importante.

Por lo tanto, valorar la cultura de seguri- dad proporciona a una organización un entendimiento básico relacionado con percepciones y actitudes de sus gerentes y personal. Las medidas de cultura de seguridad pueden ser usadas como herramientas de diagnóstico para identificar áreas para mejorar. Una evaluación en este aspecto ayuda a una organización a determinar áreas consideradas más problemáticas que otras; esta valoración puede proporcionar a una organización material para análisis de "causa/raíz" y generar ideas del propio personal involucrado hacia el mejoramiento. De esta forma, la evaluación en sí misma puede ser manejada como una intervención de seguridad del paciente (27).

Diversos estudios han analizado la cultura de seguridad en la atención de los pacientes; los resultados encontrados desde la percepción del personal de enfermería son los siguientes: En el estudio de Saturno et al. (28), el clima de seguridad global fue evaluado con una media de 7, no identificándose ninguna dimensión como fortaleza, siendo las dimensiones con puntajes más altos: trabajo en equipo intraunidad, acciones de dirección/ supervisión y cambios de turno y transición entre los servicios, mientras que las áreas identificadas de mejora continua fueron: trabajo en equipo entre unidades, retroalimentación y comunicación sobre errores y apoyo de la gerencia. El mayor porcentaje $(77,8 \%)$ no notificó ningún evento adverso.

Barbosa et al. (29) identificaron fortalezas en: "preocupación por los errores que se cometen" y "trato con respeto", mientras que las de mejora continua: "preocupación que se genera cuando se presenta un evento adverso", "no se recibe apoyo de otras áreas" y "los errores utilizados en contra de los involucrados". En relación al grado de seguridad percibida, sólo el 48,45\% la calificó como excelente-buena y el 73,48\% no realizó ningún reporte de evento adverso.

Fajardo-Dolci et al. (30) reportaron en su estudio una percepción global positiva de $49 \%$. La dimensión mejor evaluada fue el aprendizaje organizacional y la dimensión 
percibida más baja fue la respuesta no punitiva al error. El 46\% no informa eventos adversos. Mientras que en el estudio de Gutiérrez et al. (31) ninguna dimensión alcanzó un porcentaje de respuestas positivas superior al $75 \%$. Las dimensiones mejor evaluadas por el personal de enfermería fueron: el nivel de estrés, las relaciones con los compañeros y el ambiente en el lugar de trabajo, mientras que las oportunidades hacia la mejora continua correspondieron a la organización, dirección del servicio y del hospital, las condiciones de trabajo y el clima de seguridad.

Skodová et al. (32) reportaron una media de 6,57 de percepción global hacia la cultura de seguridad del paciente, no se encontró ninguna dimensión como fortaleza. La dimensión mejor valorada es el trabajo en equipo. Las dimensiones hacia la mejora continua: soporte desde la gerencia/dirección para la seguridad del paciente, dotación del personal, respuesta no punitiva al error, comunicación y feed-back sobre el error $\mathrm{y}$ comunicación abierta dentro del servicio y con los superiores. El 9,8\% notifica algún evento adverso.

Objetivo: Identificar las áreas de fortaleza y oportunidad de mejora continua, percibidas por el personal de enfermería en relación a la cultura de seguridad en la atención de los pacientes en un Hospital General del Sistema de Salud en Tampico, Tamaulipas, México.

\section{MATERIAL Y MÉTODOS}

Estudio cuantitativo y descriptivo con diseño transversal, fue realizado en el Hospital General "Dr. Carlos Canseco" de la Secretaría de Salud, en Tampico, Tamaulipas, México. La población se conformó por 472 enfermeras. El tipo de muestreo fue probabilístico estratificado proporcional, la variable de estratificación fue el turno, quedando una muestra de 195, conformado en: 41 en el turno matutino, 35 en el turno vespertino, 61 en el turno nocturno y 58 en el turno de jornada acumulada. Los criterios de inclusión: personal de enfermería laborando en área operativa, con antigüedad de más de 6 meses.

El presente estudio respetó los lineamientos éticos del reglamento de la Ley General de Salud en materia de investigación de México (Secretaría de Salubridad, 1987) (33). Se consideraron los siguientes artículos 13, 14 fracciones I, V, VII y VIII, artículo 18 y 21 , fracción VII y artículo 21 fracciones I, II, V, VIII. Se obtuvo el dictamen de la comisión de Investigación y Ética de la Facultad de Enfermería de Tampico de la Universidad Autónoma de Tamaulipas, así como la autorización de la institución y el consentimiento informado de los participantes.

El instrumento utilizado para el análisis de la cultura de la seguridad fue un indicador tipo encuesta de la Agency for Healthcare Research and Quality-AHQR, validada al contexto de español por el Ministerio de Sanidad y Consumo de Madrid, como Cuestionario sobre seguridad de los pacientes, versión española del Hospital Survey on Patient Safety, el cual está compuesto de tres apartados con doce dimensiones y 42 ítemes. El apartado A, resultados de la cultura de seguridad con dos dimensiones : "frecuencia de eventos notificados "y "percepción de la seguridad"; el apartado B, cultura de la seguridad a nivel de unidad/servicio con ocho dimensiones: "expectativas y acciones de la dirección/supervisión/servicios para favorecer la seguridad"; "aprendizaje organizacional/mejora continua"; "trabajo en equipo en la unidad/servicio"; "franqueza de la comunicación"; "feedback y comunicación sobre los errores"; "respuesta no punitiva a los errores"; "dotación de personal" y "apoyo de la gerencia del hospital en la seguridad del paciente", y el apartado C, cultura de la seguridad a nivel de todo el hospital con dos dimensiones: "trabajo en equipo entre unidades/servicios" y "problemas en cambios de turno y transiciones entre servicios/unidades". Además contiene una escala de 0 al 10 (ítem 43) para evaluar el clima de 
seguridad percibido, 9 preguntas de información complementaria personal y profesional y una pregunta abierta en donde se solicita el número de eventos reportados en el último año. Para verificar la consistencia interna del instrumento se aplico Alpha de Cronbach, la cual reporto 0.84 para la escala general, que de acuerdo a su valor se considera excelente, lo cual permite tener la confiabilidad en la medición de la variable del estudio (34).

Para identificar las áreas de fortaleza y mejora continua que conforman el análisis de la cultura de seguridad, se tuvo en cuenta las indicaciones de la AHRQ en el sentido de clasificar una dimensión como fortaleza cuando el porcentaje de respuestas positivas supera el $75 \%$. El cuestionario contiene preguntas formuladas positivamente y otras negativamente (ítemes: $5,7,8,10,12,14,16$, $17,21,22,24,25,27,28,29,31,33$ y 39$)$. En el análisis de frecuencias inicial por ítemes, se mantuvieron las opciones de respuesta originales, pero para el análisis global por dimensiones se invirtieron las preguntas que estaban formuladas en sentido negativo para facilitar el análisis.

De forma general las respuestas del cuestionario se recodificaron en tres categorías: negativo las respuestas "muy en desacuerdo, en desacuerdo, nunca y raramente", neutral las respuestas "ni de acuerdo, ni en desacuerdo y a veces" y positivo las respuestas "de acuerdo, muy de acuerdo, casi siempre y siempre". Con esta codificación se calcularon las frecuencias relativas de cada categoría, tanto para cada uno de los ítemes como del indicador compuesto de cada dimensión. Los indicadores compuestos para cada una de las dimensiones de la escala se calcularon aplicando la siguiente fórmula: $\sum$ número de respuestas positivas en los ítemes de una dimensión entre el número de respuestas totales en los ítemes de una dimensión.

Para clasificar un ítem o una dimensión como fortaleza se emplearon los siguientes criterios alternativos: $\geq$ a $75 \%$ de respuestas positivas ("de acuerdo/ muy de acuerdo" o "casi siempre/siempre") a preguntas formuladas en positivo; $\geq$ a $75 \%$ de respuestas negativas ("en desacuerdo/muy en desacuerdo" o "nunca/raramente") a preguntas formuladas en negativo. Para clasificar un ítem ó una dimensión como debilidad u oportunidad de mejora se emplearon los siguientes criterios alternativos: $\geq$ a $50 \%$ de respuestas negativas ("en desacuerdo/muy en desacuerdo" o "raramente/ nunca") a preguntas formuladas en positivo; $\geq$ a $50 \%$ de respuestas positivas ("de acuerdo/ muy de acuerdo" o "casi siempre/ siempre") a preguntas formuladas en negativo.

Para calificar el clima de seguridad se consideró el ítem 43, para lo cual se determinó la media de respuesta. Además de este ítem, el número de eventos notificados (ítem 48) y las dimensiones "frecuencia de eventos notificados" y "percepción de seguridad", se consideraron medidas de resultado del clima de seguridad.

Las variables de información complementaria se agruparon para su análisis estadístico, determinándose su frecuencia y proporción.

\section{RESULTADOS}

En relación a la información complementaria que proporcionaron los participantes, se identificó que el mayor porcentaje de acuerdo a la edad correspondió al grupo de $48-58$ años con $81(41,5 \%)$, el sexo de los participantes estuvo representado en su mayoría por mujeres $92 \%$, versus $8,2 \%$ de hombres. El mayor porcentaje se ubicó en el turno nocturno $61(31,3 \%)$, de acuerdo a la categoría laboral, las licenciadas(os) en enfermería son 68 (34,9\%); enfermeras generales $55(28,2 \%)$; enfermeras especialistas $53(27,2 \%)$ y auxiliares de enfermería $19(9,7 \%)$. El personal de enfermería que se encuentran laborando en pediatría fue el de mayor participación con 42 (21,5\%), segui- 
do del personal de medicina interna con 34 $(17,4 \%)$, urgencias con $29(14,9 \%)$, obstetricia y ginecología con $24(12,3 \%)$ y cirugía general mujeres con $21(10,8 \%)$; cirugía general hombres $20(10,3 \%)$; UCI $15(7,7 \%)$; hemodiálisis $6(3,1 \%)$ y oncología $4(2 \%)$. En relación al año de inicio de ejercicio profesional y laboral dentro de la institución 67 $(34,4 \%)$ empezaron a ejercer como enfermera entre 1981-1990, seguido de 57 (29,2\%), que empezó a ejercer en la última década. El $100 \%$ del personal de enfermería encuestado labora de manera directa con el paciente y cubre una jornada de 40 horas semanales.

En la Gráfica 1 se aprecian los porcentajes obtenidos de respuestas positivas posterior a la recodificación de los ítemes negativos a positivos de cada una de las dimensiones que conforman el instrumento. Las dimensiones que obtuvieron porcentajes superiores al $75 \%$, por lo cual se perciben como fortalezas, fueron: aprendizaje organizacional y trabajo en equipo en la unidad/servicio, mientras que las dimensiones evaluadas con menos del $50 \%$ de respuestas positivas correspondieron a: dotación de personal; respuesta no punitiva a los errores; apoyo de la gerencia del hospital; percepción de seguridad; problemas en los cambios de turno/servicio y franqueza en la comunicación, lo cual las identifica como áreas con oportunidad hacia la mejora continua.

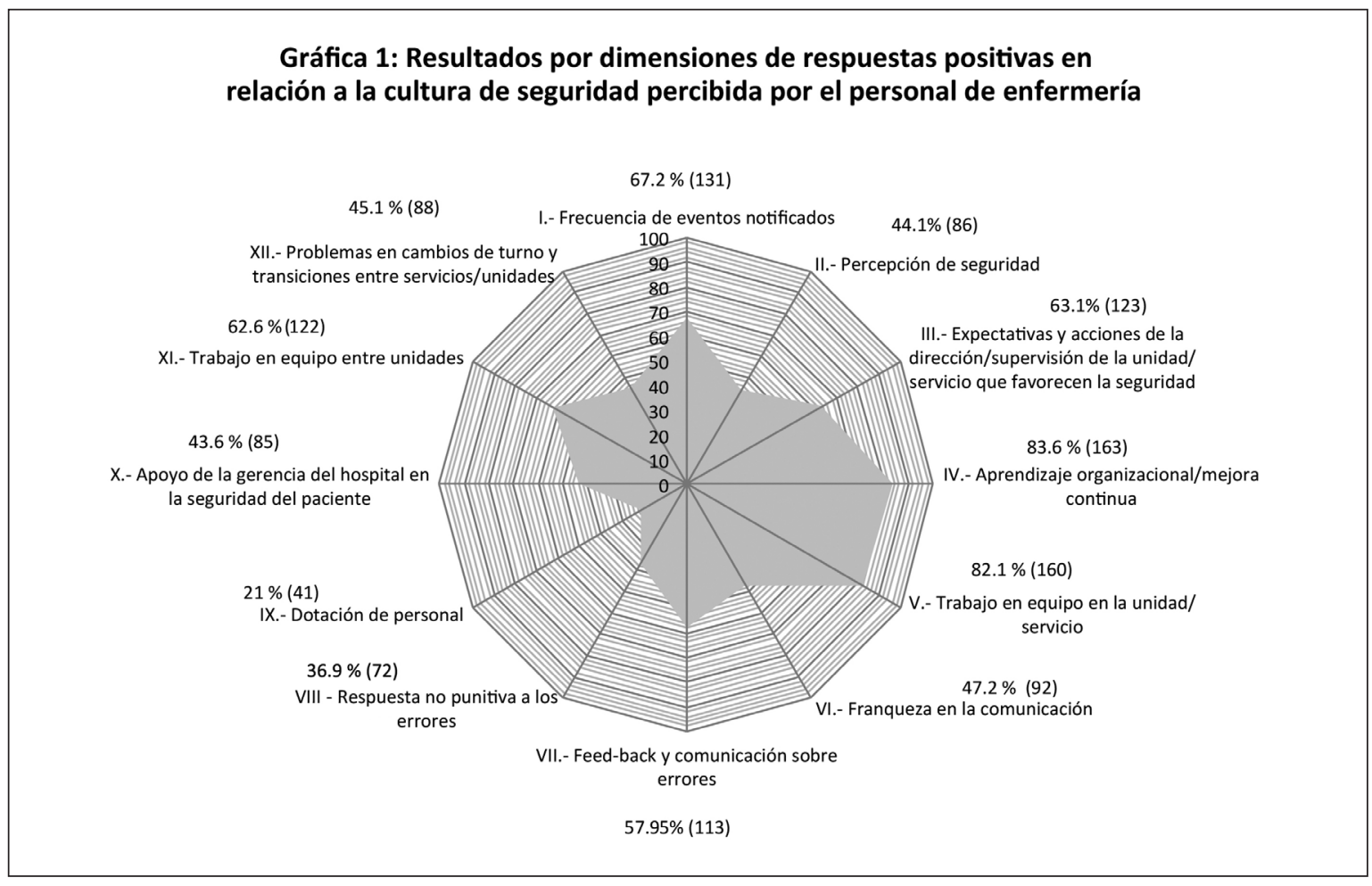

En la Tabla 1 se presentan los datos estadísticos en relación al análisis por ítemes fuertes y mejor evaluados, los cuales se incluyen en las dimensiones de: aprendizaje organizacional, trabajo en equipo, frecuencia de eventos notificados, percepción de la seguridad y trabajo entre unidades.
La Tabla 2 presenta los datos relativos a los ítemes con oportunidad hacia la mejora continua, los cuales se englobaron en las dimensiones de: dotación de personal, respuesta no punitiva a los errores, apoyo de la gerencia del hospital en la seguridad del paciente, feed-back y comunicación de erro- 
Tabla 1. Ítemes con mayor número de respuestas positivas evaluadas por el personal de enfermería.

\begin{tabular}{|c|c|c|c|}
\hline Dimensión & Ítem & fr & $\%$ \\
\hline IV: Aprendizaje organizacional/mejora continua & $\begin{array}{l}\text { Cuando se detecta algún fallo en la atención al paciente } \\
\text { se llevan a cabo las medidas apropiadas para evitar que } \\
\text { ocurran de nuevo }\end{array}$ & 153 & 78 \\
\hline V: Trabajo en equipo en la unidad /servicio & El personal se apoya mutuamente & 150 & 77 \\
\hline V: Trabajo en equipo en la unidad /servicio & En esta unidad nos tratamos con respeto & 149 & 76 \\
\hline IV: Aprendizaje organizacional/mejora continua & $\begin{array}{l}\text { Tenemos actividades a mejorar la seguridad del pacien- } \\
\text { te }\end{array}$ & 147 & 75 \\
\hline V: Trabajo en equipo en la unidad /servicio & $\begin{array}{l}\text { Cuando tenemos mucho trabajo, colaboramos todos } \\
\text { como un equipo para poder terminarlo }\end{array}$ & 147 & 75 \\
\hline V: Trabajo en equipo en la unidad /servicio & $\begin{array}{l}\text { Cuando alguien está sobrecargado de trabajo, suele } \\
\text { encontrar ayuda en sus compañeros }\end{array}$ & 137 & 70 \\
\hline II: Percepción global de seguridad & $\begin{array}{l}\text { Nuestros procedimientos y medios de trabajo son bue- } \\
\text { nos para evitar errores en la asistencia }\end{array}$ & 134 & 69 \\
\hline IV: Aprendizaje organizacional/mejora continua & $\begin{array}{l}\text { Los cambios que hacemos para mejorar la seguridad del } \\
\text { paciente se evalúan para comprobar su efectividad }\end{array}$ & 128 & 66 \\
\hline I: Frecuencia de eventos notificados & $\begin{array}{l}\text { Se notifican los errores que son descubiertos y corregi- } \\
\text { dos antes de afectar al paciente }\end{array}$ & 125 & 65 \\
\hline XI: Trabajo en equipo entre unidades & $\begin{array}{l}\text { Los servicios/ unidades trabajan de forma coordinada } \\
\text { entre sí para proporcionar la mejor atención posible a } \\
\text { los pacientes }\end{array}$ & 123 & 64 \\
\hline
\end{tabular}

Fuente: AHQR

Tabla 2. Ítemes con menos respuestas positivas evaluadas por el personal de enfermería.

\begin{tabular}{l} 
Dimensión \\
\hline IX: Dotación de personal \\
VIII: Respuesta no punitiva a los errores \\
IX: Dotación de personal \\
X: Apoyo de la gerencia del hospital en la segu- \\
ridad del paciente
\end{tabular}

II: Percepción global de seguridad

IX: Dotación de personal

XII: Problemas en cambios de turno y transiciones entre servicios/unidades

VII: Feed-back y comunicación sobre errores

IX: Dotación de personal

XI: Trabajo en equipo entre unidades

\begin{tabular}{|c|}
\hline Ítem \\
\hline Hay suficiente personal para afrontar la carga de trabajo \\
\hline $\begin{array}{l}\text { Cuando se comete un error, el personal teme que eso } \\
\text { quede en el expediente }\end{array}$ \\
\hline $\begin{array}{l}\text { A veces no se puede proporcionar la mejor atención al } \\
\text { paciente porque la jornada laboral es agotadora }\end{array}$ \\
\hline $\begin{array}{l}\text { La gerencia/dirección del hospital sólo parece interesarse } \\
\text { por la seguridad del paciente cuando ya ha ocurrido } \\
\text { algún suceso adverso en un paciente }\end{array}$ \\
\hline No se producen más fallos por casualidad \\
\hline $\begin{array}{l}\text { Trabajamos bajo presión para realizar demasiadas cosas } \\
\text { demasiado deprisa }\end{array}$ \\
\hline $\begin{array}{l}\text { Surgen problemas en la atención de los pacientes como } \\
\text { consecuencia de los cambios de turno }\end{array}$ \\
\hline $\begin{array}{l}\text { Cuando notificamos algún incidente, nos informan sobre } \\
\text { qué tipo de actuaciones se han llevado a cabo }\end{array}$ \\
\hline $\begin{array}{l}\text { En ocasiones no se presta la mejor atención al paciente } \\
\text { porque hay demasiados sustitutos o personal temporal }\end{array}$ \\
\hline $\begin{array}{l}\text { Las diferentes unidades del hospital no se coordinan bien } \\
\text { entre ellas }\end{array}$ \\
\hline
\end{tabular}

\begin{tabular}{l|l} 
fr & $\%$ \\
\hline 32 & 16 \\
36 & 18 \\
48 & 25 \\
55 & 28 \\
56 & 29 \\
62 & 32 \\
68 & 35 \\
68 & 35 \\
73 & 37 \\
73 & 37 \\
\hline
\end{tabular}


res, trabajo en equipo entre unidades y problemas en cambios de turno y transiciones entre servicios/unidades.

El clima de seguridad obtuvo una media de 7,35 en una escala del 0 al 10, la percepción de seguridad fue percibida como una dimensión hacia la mejora continua con 86 $(44,1 \%)$; mientras que la dimensión de frecuencia de eventos notificados obtuvo 131 $(67,2 \%)$, en relación a la frecuencia de eventos notificados 156 participantes reportaron 0 eventos con un 80\%, 17 reportó 1 evento con un $8,7 \%, 13$ con 2 eventos reportados con un $6,7 \%, 2$ con 3 con un $1,0 \%$ y 3 con 5 eventos reportados con un 1,5\%. La percepción global del indicador de cultura de la seguridad fue percibido con un $54,53 \%$ de respuestas positivas.

\section{DISCUSIÓN Y CONCLUSIÓN}

El indicador global de la cultura de la seguridad no fue percibido como fortaleza por el personal de enfermería con un 54,53\%, este resultado fue similar a lo reportado en otros estudios (28, 30-32), en donde ninguna dimensión del indicador de cultura de la seguridad en la atención de los pacientes fue percibida por el personal participante como fortaleza, lo cual indica que se debe fortalecer dentro de la organización la cultura de seguridad, atendiendo las áreas identificadas como oportunidad hacia la mejora continua y continuar trabajando en las dimensiones representadas como fortalezas.

La dimensión que mostraron una tendencia de fortaleza en este estudio correspondió al trabajo en equipo, que explora aspectos relacionados con el apoyo, respeto y solidaridad del personal para trabajar en situación normal y de sobredemanda con un 82,1\%, similar a lo reportado por Saturno et al. (28), en donde el "trabajo en equipo" fue de las dimensiones mejores evaluadas con un $71,8 \%$; al realizar el análisis por ítemes, se encontró que "el personal se apoya mutuamente" presentó un $78,2 \%$ de respuestas positivas; en este mismo estudio el trabajo en equipo intraunidad fue bien evaluado por el personal de enfermería con un $73,8 \%$.

Barbosa et al. (29) reportó en su estudio un $75,77 \%$ de respuestas positivas hacia "el trato con respeto", mientras que los estudios de Gutiérrez et al. (31) reportaron un $61,56 \%$ para ambiente en el lugar de trabajo y $62,5 \%$ para las relaciones con los compañeros de trabajo, y Skodová et al. (32) reportó un $66,95 \%$ de respuestas positivas para la dimensión del trabajo en equipo, lo que indica que las relaciones interpersonales intraunidad tales como el respeto, la ayuda mutua, la colaboración y la ayuda son percibidas como un comportamiento positivo del personal de enfermería en cada uno de los servicios en los que laboran.

La dimensión de aprendizaje organizacional/mejora continua, que explora aspectos relacionados a las actividades para mejorar la seguridad, medidas apropiadas para evitar que ocurran nuevamente riesgos en los pacientes, así como la evaluación en los cambios relacionados con la seguridad fue percibida como fortaleza con un $83,6 \%$, encontrándose una similitud a los estudios de Saturno et al. (28) con una percepción global del $61,8 \%$, y por parte del personal de enfermería con un $64,4 \%$. En el análisis por ítemes "cuando se detecta algún fallo en la atención al paciente se llevan a cabo las medidas apropiadas para evitar que ocurra de nuevo" con un 78\%; al igual Barbosa et al. (29) quienes reportaron en su estudio un $78,35 \%$ de respuestas positivas por la "preocupación por los errores que se cometen". Semejante fue lo reportado por Skodovà et al. (32) con una evaluación del $71,9 \%$ para esta dimensión al igual que Fajardo-Dolci et al. (30) encontraron que el "aprendizaje organizacional" fue de las dimensiones mejor evaluadas.

En relación a la dimensión de frecuencia de eventos notificados que indaga aspectos relacionados con el reporte acerca de erro- 
res que pudieran haber lesionado o no al paciente, este estudio reporta un $67,2 \%$, a diferencia de otros estudios que han reportado porcentajes de $48,97 \%$ (29) y $77,8 \%$ (28) respuestas negativas para esta dimensión.

La dimensión de las expectativas y acciones de la dirección/supervisión de la unidad/ servicio que favorecen la seguridad, que indaga las acciones de la dirección y supervisión y analiza aspectos concernientes al compromiso del personal de enfermería que gerencia el cuidado que presta fue evaluada con un $63,1 \%$, similar al trabajo de Saturno et al. (28) en donde reportaron esta dimensión como una de las mejores evaluadas, desde la percepción del personal de enfermería con un $61,8 \%$.

Concerniente a las áreas de oportunidad hacia la mejora continua en este estudio se encontraron seis dimensiones; la dimensión peor evaluada correspondió a la "dotación de personal", que explora aspectos de las cargas de trabajo laboral en relación a la cantidad de personal para brindar una atención con seguridad, con un 21\%; contrario al 51,5\% reportado por Skodová et al. (32); en relación a la dimensión de la "respuesta no punitiva a los errores" que indaga aspectos relativos a la percepción que se tiene cuando se comete algún error y las consecuencias que ello pueda tener fue evaluada con un $36,9 \%$, similar a lo reportado por Fajardo et al. (30) con un 26\%, en contraste con Skodová et al. (32) con un 51,5\%; en la dimensión "franqueza de la comunicación" que estudia la libertad que tiene el personal para tratar las situaciones que condicionaron un evento adverso se reportó un $47,2 \%$, porcentaje superior a lo reportado por Skodová et al. (32) con un $36,06 \%$.

La dimensión de "apoyo de la gerencia del hospital" en la seguridad que averigua el compromiso de la dirección con los aspectos relacionados con el clima de seguridad y el compromiso que asumen ante cualquier hecho que pudiera condicionar situaciones de inseguridad para los pacientes, se encontró con un $43,6 \%$, a diferencia de lo reportado por Gutiérrez et al. (31) con un 27,5\% y contrario al $64,4 \%$ descrito por Saturno et al. (28) y al 71,9\% de Skodová et al.(32). Referente a la dimensión en donde se evaluaron aspectos relativos a la percepción del clima de la seguridad se reportó un $44,1 \%$, este porcentaje se encuentra dentro del rango reportado por otros estudios, $33,8 \%$ a 50,1\% (28-31); la dimensión de feed-back y comunicación sobre errores con un 57,9\% a diferencia de lo reportado por Skodová et al. (32) con un $36,9 \%$.

La dimensión del trabajo en equipo entre unidades que explora el comportamiento de seguridad en los enlaces de la atención de los pacientes entre los servicios de hospitalización, reporta un $62,6 \%$, en contraste al reporte de Saturno et al. (28) con un 73,8\% de respuestas negativas para esta dimensión.

Este estudio evaluó el clima de seguridad con una media de 7,35, similar a Saturno et al. (28) con 7 y a diferencia del reporte de Skodová et al. (32) con una media de 6,5; en el estudio de Barbosa et al. (29) en donde el grado de seguridad percibida sólo el 48,45\% la calificó como excelente-buena y el 27,84\% la refiere aceptable; para Fajardo et al. (30) la percepción global de seguridad fue de $49 \%$. Los eventos adversos en el $80 \%$ de este estudio no se notifican, todos los estudios revisados mostraron la misma tendencia (28-32).

Las conclusiones que se determinan en el estudio muestran que el análisis del indicador utilizado permitió identificar la percepción global del personal de enfermería hacia la seguridad en la atención del paciente en el Hospital General "Dr. Carlos Canseco" en Tampico, Tamaulipas, identificando seis áreas o dimensiones problemáticas con oportunidad hacia la mejora continua; así como dos dimensiones fuertes dentro de la cultura de seguridad en las que se brinda el cuidado de enfermería hacia los pacientes. 


\section{REFERENCIAS}

1. Aranaz JM, Aibar C, Galán A, Limón R, Requena J, Álvarez EE, et al. La asistencia sanitaria como factor de riesgo: los efectos adversos ligados a la práctica clínica. Gaceta Sanitaria. 2006; 20(1):41-7.

2. Aranaz JM, Agra Y. La cultura de la seguridad del paciente: del pasado al futuro en cuatro tiempos. Medicina Clínica (Barcelona). 2010; 135(1): 1-2.

3. Acevedo G, Castañeda H, Garza R, González JF, Meléndez MC, Patiño ML, Pineda MM, Rangel S, Acevedo GJ, Aguilera A. Importancia de la satisfacción como indicador de la calidad en enfermería. 1a ed. México: Ed. UNIVERSUM; 2008: $1-32$.

4. Cometto MC, Gómez PF, Dal Sasso GT, Zárate RA, De Bortoli SH, Falconí C. Enfermería y seguridad de los pacientes. $1^{\text {a }}$ ed. Washington, D.C: Ed. Organización Panamericana de la Salud; 2011: 1-17.

5. Organización Mundial de la Salud [Internet]. Seguridad del paciente. La investigación en seguridad del paciente. Ginebra: OMS [citado 13 junio 2010]. Disponible en: http://www.who.int/patientsafety/research/es/

6. Schimmel EM. The hazards of hospitalization. Quality Safety Health Care. 2003; 12: 58-64.

7. Brennan TA, Leape LL, Laird NM, Hebert L, Localio AR, Lawthers AG, et al. Incidence of adverse events and negligence in hospitalized patients. Results of the Harvard Medical Practice Study I. New England Journal Medicine. 1991; 324: 370-376.

8. Gea-Velázquez MT, Aranaz-Andrés JM. Eventos adversos asociados a la asistencia del paciente pluripatológico ingresado en hospitales de crónicos. Medicina Clínica (Barcelona). 2010; 135(2): 17-23.

9. Muiño A, Jiménez B, Pinilla B, Durán E, Cabrera J, Rodríguez P. Seguridad del pa- ciente. Anales de Medicina Interna (Madrid). 2007; 24(12): 602-606.

10. Kohn L, Corrigan JM, Donaldson MS. Committee on Quality of Health Care in America. To Err Is Human: Building a safer health system. Washington, DC: Institute of Medicine, National Academies Press; 2000: 312.

11. World Health Organization. World Alliance for Patient Safety: Forward programme. Geneva: WHO Library; 2004.

12. Astolfo F. La seguridad clínica de los pacientes: entendiendo el problema. Colombia Médica. 2005; 36: 130-133.

13. The National Quality Forum: Safe Practices for Better Healthcare. A Consensus report. Washington: NQF; 2003.

14. The National Quality Forum. Safe Practices for Better Health Care, 2006 Update. Washington: NQF; 2007 [citado 6 septiembre 2011]. Disponible en: http:// www.csen.com/err.pdf

15. Cooper, MD. Towards a model of Safety Culture. An abridged version published in Safety Science. 2000; 36: 111-136.

16. Pizzi L, Goldfarb N, Nash D. Promoting a Culture of Safety. Thomas Jefferson University School of Medicine and Office of Health Policy and Clinical Outcomes. 2001 [citado 10 septiembre 2011]. Disponible en: http://www.ahcpr.gov/clinic/ ptsafety/chap40.htm

17. Hewison AR. Organizational culture: a useful concept for nurse manager. Journal of Nursing Administration. 1996; 4: 3-9.

18. Institute of Medicine. Cross the Quality Chasm: A New Health System for the $21^{\text {st }}$ Century. The National Academic Press. Washington DC; 2001.

19. Hemman EA. Creating health care cultures of patient safety. Journal of Nursing Administration. 2002; 32: 419-428.

20. Institute of Medicine. Keeping Patient Safe: Transforming the Work Environment of Nurses. The National Academic Press. Washington DC; 2004. 
21. Feng X, Bobay K, Weiss M. Patient safety culture in nursing: a dimensional concept analysis. Journal of Advanced Nursing. 2008; 63(3): 310-319.

22. Helmreich RL, Merritt AA. Culture at work in aviation and medicine: national organizational, and professional influences. Aldershot; BBrookfield, VT,USA: Ashgate; 1998.

23. Sorra JS, Nieva VF. Hospital Survey on Patient Safety Culture. Rockville, MD: Agency for Health Care research and Quality. 2004 [citado 1 agosto 2011]. Hallado en: http://www.ahrq.gov/qual/ hospculture/hospcult.pdf

24. León CA. La seguridad del paciente, una responsabilidad en el acto del cuidado. Revista Cubana de Enfermería. 2006; 22(3): 1-7.

25. Seguridad del paciente. La enfermera importa. Comunicado de prensa 29 de abril 2002 [citado 12 mayo 2010]. Disponible en: http://www.icn.ch/matters_ptsafetysp.htm

26. Clancy CM, Farquhar MB, Collins BA. Patient safety in nursing practice. Journal Nursing Care Quality. 2005; 20: 193-197.

27. NievaVF, Sorra JS, Safety culture assessment: a tool for improving patient safety in healthcare organizations. Quality and Safety in Health Care. 2003; 12(supl 2): ii17-23.

28. Saturno PJ, Da Silva ZA, de OliveiraSousa SL, Fonseca YA, De Souza-Oliveira $\mathrm{AC}$, et al. Análisis de la cultura sobre seguridad del paciente en los hospitales del Sistema Nacional de Salud Español. Medicina Clínica Monografías (Barcelona).
2008; 131(3): 18-25.

29. Barbosa R, Cárdenas C, García C, More P, Muñoz O. Establecimiento de una línea de base de la cultura de la seguridad de los pacientes en un hospital universitario de Bogotá. Revista Iberoamericana de psicología: ciencia y tecnología. 2008; (1): 19-28.

30. Fajardo-Dolci G, Rodríguez-Suárez J, Arboleya-Casanova H, Rojano- Fernández C, Hernández-Torres F, Santa CruzVarela J. Cultura sobre seguridad del paciente en profesionales de la salud. Cirugía Cirujanos. 2010; 78(6): 522-527.

31. Gutiérrez-Cía I, Merino de Cos P, Yáñez A, Obon-Azuara B, Alonso-Ovies A, Martin-Delgado M, et al. Percepción en la cultura de seguridad en los servicios de medicina intensiva españoles. Medicina Clínica (Barcelona). 2010; 135(1): 37-44.

32. Skodová M, Velasco MJ, Fernández MA. Opinión de los profesionales sanitarios sobre seguridad del paciente en un hospital de primer nivel. Revista de Calidad Asistencial, 2011; 26(1): 33-38.

33. Estados Unidos Mexicanos. Reglamento de la Ley General de Salud en Materia de Investigación para la salud (1984). Ley General de Salud. México.

34. Cuestionario sobre seguridad de los pacientes. Versión española del Hospital Survey on Patient Safety. Madrid: Ministerio de Sanidad y Consumo [citado 1 octubre 2010]. Disponible en: http:// www.msc.es/organizacion/sns/planCalidadSNS/docs/CuestionarioSeguridadPacientes 